\title{
An All-in-One Molecule for the One-Step Synthesis of Functional Hybrid Silica Particles with Tunable Sizes
}

\author{
Julien Graffion, ${ }^{[a, b]}$ Dounia Dems, ${ }^{[a, b]}$ Mesut Demirelli, ${ }^{[a, b]}$ Thibaud Coradin, ${ }^{[a, b]}$ \\ Nicolas Delsuc, ${ }^{[c]}$ and Carole Aimé*[a,b]
}

\begin{abstract}
Spherical particles with well-defined diameters were obtained by self-assembly of trityl-based molecules. Thanks to the robustness of the organic scaffold, a variety of modifications could be covalently introduced into the network so as to stabilize the supramolecular structure by a sol-gel route. Using supramolecular chemistry, we showed that the synthesis of hybrid small molecules allowed engineering nanomaterials with tun-
\end{abstract}

able size and functionality. The use of a combination of different characterization techniques, including dynamic light scattering, cryoTEM, and solid-state NMR spectroscopy, provided careful understanding of the relationship between the molecular and supramolecular structures for further chemical engineering of supramolecular hybrid materials.

\section{Introduction}

Antisolvent precipitation is an attractive technique for the elaboration of nanoparticles (NPs) and finds applications in various fields such as electro-optics, catalysis, and nanomedicine, notably with the elaboration of imaging agents and for improving drug formulation. Alternatively, the design and synthesis of nanoscale metal-organic frameworks have provided tunable nanoobjects to serve as functional platforms. ${ }^{[1-6]}$ However, despite the size tunability of the resulting particles, their high interfacial area renders them prone to aggregation and Oswald ripening, which affect their stability. ${ }^{[7]}$ Research efforts have been devoted to the selection of stabilizers to be added to the precipitating nanoparticles. ${ }^{[8]}$ To this aim, amphiphilic diblock copolymers have been used, typically a hydrophilic poly(ethylene glycol) (PEG) block chemically linked to a hydrophobic block. ${ }^{[9]}$ Thanks to its amphiphilic properties, the polymer adsorbs at the surface of the NPs, which limits further growth or dissolution and, thus, prevents aggregation. Polyelectrolytes such as polylysine, poly(ethylene imine), and chitosan have also been used to stabilize the NPs electrostatically and sterically and to ensure a narrow size distribution. ${ }^{[10]}$ Alternatively, hybrid (organic-inorganic) materials have been intensively investigated for two decades owing to their unique properties that

[a] Sorbonne Universités, UPMC Univ Paris 06, Collège de France, UMR CNRS 7574, Laboratoire de Chimie de la Matière Condensée de Paris, Paris cedex 05, France

E-mail: carole.aime@upmc.fr www.labos.upmc.fr/lcmcp

[b] PSL Research University, 60 rue Mazarine, 75006 Paris, France

[c] Laboratoire des Biomolécules, Département de Chimie, École normale supérieure, PSL Research University, Sorbonne Universités, UPMC Univ. Paris 06, CNRS,

24 rue Lhomond, 75005 Paris, France

Supporting information and ORCID(s) from the author(s) for this article are available on the WWW under https://doi.org/10.1002/ejic.201701181. combine the hardness and stability of the inorganic phase with the flexibility and mild processing of polymer materials. ${ }^{[11,12]}$ As recently reviewed, ${ }^{[13]}$ silsesquioxane NPs have been synthesized from organotrialkoxysilane precursors by different routes, including hydrolytic ${ }^{[14-17]}$ and nonhydrolytic ${ }^{[18]}$ sol-gel routes and emulsion polymerization. ${ }^{[19-22]}$ With such processes, spherical, $^{[14,15,23-25]}$ hollow, $^{[26-30]}$ and Janus ${ }^{[31]}$ NPs have been obtained. Their surface can be easily functionalized through silanization reactions of superficial silanol groups. ${ }^{[32,33]}$ This offers a large number of possibilities to bind targeting moieties for imaging or drug-delivery systems ${ }^{[34]}$ or to enhance cell compatibility and internalization. ${ }^{[35,36]}$

Among polysilsesquioxanes, phenylsilsesquioxanes have drawn much attention, and the synthesis of $\mathrm{PhSiO}_{3 / 2}$ particles has been reported. ${ }^{[16,37]}$ In another study, Dirè et al. synthesized NPs from a series of alkyltriethoxysilane (methyl-, ethyl-, vinyl-, phenyl-, amyl-, and octyltriethoxysilane) with systematic assessment of the influence of the different organic groups on the synthesis and properties of the silsesquioxane NPs. ${ }^{[25]}$ Such molecular design allowed the doping of particles, either by physical entrapment or covalent linking, with representative examples using fluorophores such as Nile red, ${ }^{[38]}$ rhodamine $B,{ }^{[17,24,39]}$ and rhodamine $6 G .{ }^{[25]}$ Notably, for the abovecited alkyltriethoxysilanes, the formation of nanoparticles results from nucleation and growth induced by sol-gel polymerization, which is typically followed by a two-step hydrolysiscondensation process, as in the works of Dirè et al. On the other hand, the self-assembly of silsesquioxanes has been reported for the design of helical and lamellar structures with organogelator-like behavior. ${ }^{[40-42]}$

In this work, we exploit the supramolecular interaction properties of the organic moiety of organotrialkoxysilanes as the only molecular key towards nanoparticles. We use different molecular designs based on the triphenylmethane (trityl) group (Figure 1) to take advantage of this moiety to drive the forma- 


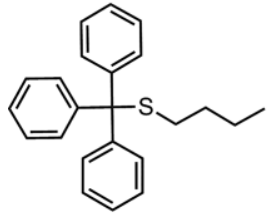

1

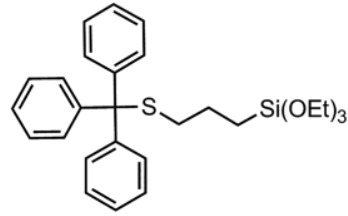

2

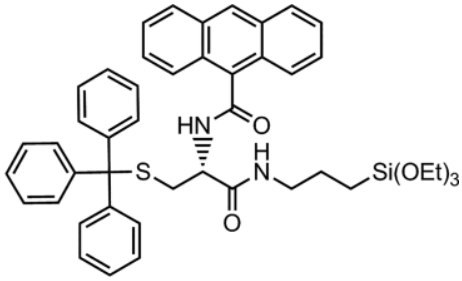

3

Figure 1. Molecular structures synthesized from an alkyl-S-trityl moiety (see compound 1), in combination with a triethoxysilane group (see compound 2), and belonging to a cysteine functionalized with an anthracene (see compound $\mathbf{3}$ ).

tion of well-defined objects. The addition of a trialkoxysilane group (see compound 2, Figure 1) aims at allowing their stabilization through sol-gel chemistry by preventing aggregation and Oswald ripening. Finally, to investigate the scope of the tolerated modifications that preserve the structure of the objects, we introduced a chiral multifunctional backbone, that is, the amino acid L-cysteine. Its thiol group was functionalized with the trityl group, as in compounds $\mathbf{1}$ and $\mathbf{2}$, and the trialkoxysilane was introduced by dicyclohexylcarbodiimide (DCC)/ $\mathrm{N}$-hydroxysuccinimide (NHS) coupling with (3-aminopropyl)triethoxysilane (APTES). A fluorescent anthracene moiety was conjugated to the amine function of the cysteine. This provided cues for functional implementation of the resulting supramolecular structures in an "all-in-one molecular structure" (see compound 3, Figure 1). The three molecules were synthesized as described in Schemes S1 and S2 (Supporting Information) and were obtained in high purity (> $95 \%$, no detectable impurities by ${ }^{1} \mathrm{H}$ NMR spectroscopy).

\section{Results and Discussion}

Antisolvent precipitation in an ethanol/water mixture was used to obtain supramolecular objects from 1. Precipitation was monitored by dynamic light scattering (DLS) in the range of 60 to $95 \%$ water and led to well-defined objects with sizes from 210 to about $100 \mathrm{~nm}$ (Figure 2a). No supramolecular structure could be detected below this range owing to the solubility of
1 in ethanol, whereas macroscopic objects sedimented above $95 \%$ water. Very interestingly, similar trends could be observed by precipitating $\mathbf{2}$ and 3, and a constant decrease in size from 60 to $80 \%$ water gave rise to well-defined objects with a mean hydrodynamic diameter above $200 \mathrm{~nm}$ at $60 \%$ (210 nm for 1, $260 \mathrm{~nm}$ for 3) and leveling around $90 \mathrm{~nm}$ from 80 to $95 \%$ water. Notably, as the water content increased, the size distribution became narrower (Table S1). Playing with the tritylated molecule concentration at a given water content could also be used to tune the size of the resulting supramolecular structures. At $90 \%$ water, upon increasing the alkoxysilane concentration from 100 to $400 \mu \mathrm{M}$, the hydrodynamic diameter of $\mathbf{2}$ steadily varied from 95 to $125 \mathrm{~nm}$ and that of $\mathbf{3}$ varied from 80 to $100 \mathrm{~nm}$ (Figure 2b). CryoTEM observations showed the formation of well-defined spherical objects in solution (Figure $2 \mathrm{~b}$, inset). Overall, the precipitation of $\mathbf{1}$ and the similar trends observed for $\mathbf{2}$ and $\mathbf{3}$ showed that the strong hydrophobic character and the nonplanar structure (one sp3 carbon atom) of the trityl group favored $\pi$-stacking interactions over a short range, and therefore, the trityl group is particularly interesting for antisolvent precipitation to obtain well-defined spherical objects. Given that trityl assembly is the driving force for the formation of spherical particles, its scaffolding role is strong enough to ensure particle formation upon the addition of even large organic molecules. Interestingly, neither the alkoxysilane nor the chiral center and anthracene moiety seemed to play an important role in obtaining well-defined nanostructures, as $\mathbf{1}, \mathbf{2}$, and
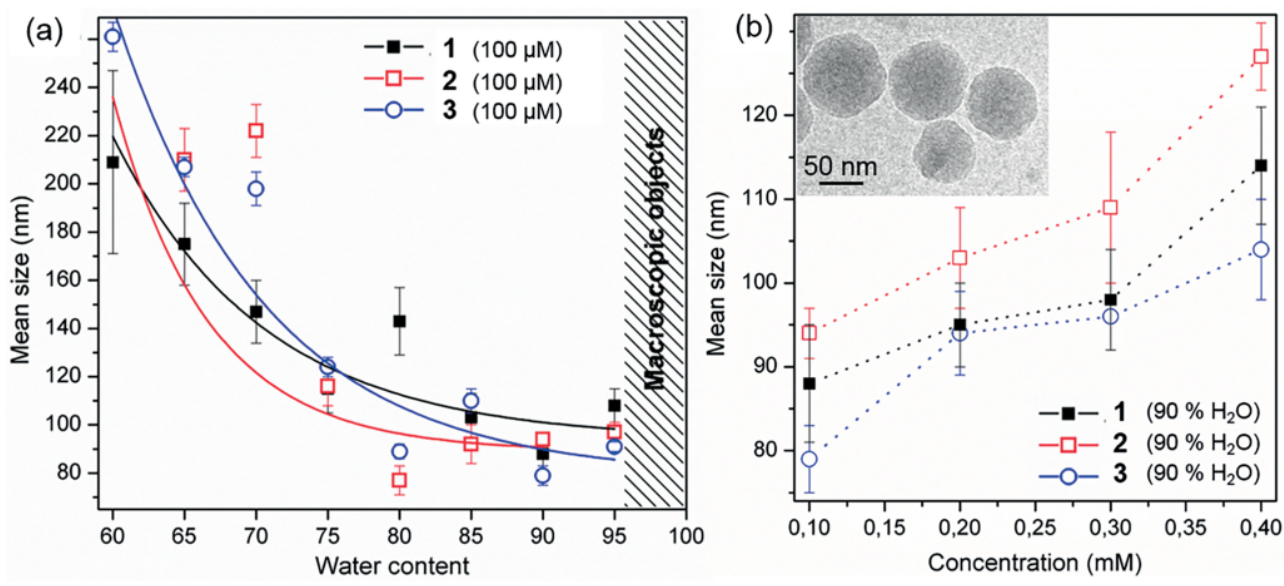

Figure 2. DLS of the supramolecular structures of $\mathbf{1}, \mathbf{2}$, and $\mathbf{3}$ as a function of (a) water content (100 $\mu \mathrm{M})$, and (b) concentration ( $90 \%$ water). The inset shows the cryoTEM image of $\mathbf{3}(200 \mu \mathrm{M}, 90 \%$ water). 
3 behaved similarly. In the presence of a chiral center, the preservation of a chiral environment within the hybrid particle could be ascertained by circular dichroism spectropolarimetry (Figure S3). Because this supramolecular chirality is expressed without modifying any of the previously described characteristics of the nanomaterials, this process is very tolerant to a wide range of modifications.

The introduction of the alkoxysilane group may offer a way to stabilize the supramolecular particles of $\mathbf{2}$ and $\mathbf{3}$. Indeed, the size of objects from $\mathbf{1}$ increased progressively up to $(633 \pm 40) \mathrm{nm}$ after 1 month (Figure 3a). In the case of precursors $\mathbf{2}$ and 3, after nanoparticle formation, a sol-gel route was used by placing the samples under ammonia vapors for $24 \mathrm{~h}$ to catalyze their hydrolysis-condensation under basic conditions. For example, in the case of $\mathbf{3}$, after this treatment, the size of the spheres leveled out at $127 \pm 3 \mathrm{~nm}$ after 1 day, whereas in the absence of ammonia, stable spherical structures exhibiting a size of $170 \pm 7 \mathrm{~nm}$ could be obtained after 3 days. This showed, one, the role played by the alkoxysilane moiety in stabilizing the final structures and, two, that ammonia was not mandatory to stabilize $\mathbf{3}$ but that it sped up the kinetics of the sol-gel process. TEM observations after stabilization of $\mathbf{2}$ and $\mathbf{3}$ revealed that the spherical structures were preserved (Figure $3 \mathrm{~b})$. In terms of chemical structure, the hydrolysis-condensation of the alkoxysilane and the formation of the siloxane shell at the surface were ascertained by $\zeta$-potential measurements and solid-state ${ }^{29} \mathrm{Si}$ NMR spectroscopy. Stabilized particles from $\mathbf{2}$ and $\mathbf{3}$ showed negative $\zeta$ potentials, which indicated the presence of silanolate groups, resulting from hydrolysis of the ethoxysilane groups, on their surface (Table S2). Probing the silicon chemical environment of $\mathbf{3}$ after stabilization by ${ }^{29} \mathrm{Si}$ NMR spectroscopy revealed broad signals around $-45.4,-57.1$, and $-66.8 \mathrm{ppm}$ that could be attributed to $\mathrm{R}-\mathrm{Si}(\mathrm{OEt})_{3}, \mathrm{R}-\mathrm{Si}(\mathrm{OSi})_{2}(\mathrm{OH})(\mathrm{T} 2)$, and $\mathrm{R}-\mathrm{Si}(\mathrm{OSi})_{3}(\mathrm{~T} 3)$, respectively; these signals confirmed partial alkoxysilane condensation (Figure S1). Further investigations by FTIR spectroscopy showed that the Si-O deformation region $\left(900-1145 \mathrm{~cm}^{-1}\right.$ ) did not change upon comparing $\mathbf{3}$ as the molecular precursor and as stabilized particles (Figure S2). In addition, no condensed siloxane bond $\left[v_{\text {as }}(\mathrm{SiOSi})\right]$ could be identified owing to their low concentration, which was restricted to the surface. These results provided evidence that the precursor remained intact within the nanoparticles owing to the hydrophobic character of the core, which prevented internal diffusion of water and ammonia. Further investigations by using powder X-ray diffraction (PXRD) showed the presence of amorphous silica, which could be expected given the huge number of molecules involved in the formation of the $100 \mathrm{~nm}$ diameter particles (Figure S4).

As a means of imparting the particle with functionality, a fluorescent probe, namely, an anthracene moiety, was introduced to the molecular design (see compound 3 ). Covalent conjugation of the anthracene probe prevented leaching of the fluorophore. Figure $4 a$ shows the emission spectra of $\mathbf{3}$ upon increasing the water content up to $90 \%$. From 0 to $50 \%$ water, emission of the anthracene moiety (well-defined components, maximum intensity at $407 \mathrm{~nm}$ ) indicates that the anthracene moiety exists as a monomer in solution. From 60 to $90 \%$ water, the emission spectra drastically broaden and become redshifted (maximum intensity at $\approx 470 \mathrm{~nm}$ ). These changes can be attributed to the transition of soluble $\mathbf{3}$ into its precipitated state, which is in good agreement with the DLS measurements, by which no supramolecular structure could be detected below $60 \%$ water. As expected, the excitation spectrum was also slightly redshifted $(7 \mathrm{~nm})$ upon increasing the water content (Figure S5a).

Given that $\mathbf{3}$ was shown to be an efficient multifunctional scaffold to form hybrid nanoparticles with easily tunable diameters, we investigated the possibility to increase the functionality of the hybrid nanoparticles by physical encapsulation. As proof of concept, the encapsulation ability of the hybrid particles was assessed by the addition of rhodamine B (rhod-B) during the precipitation of $\mathbf{3}$. Rhod-B was chosen to obtain insight into the impact of adding sizeable molecules on the stability and size of the hybrid particles. In addition, rhod-B was selected because it provides a straightforward read out of the encapsulation by using Förster resonance energy transfer (FRET) with the anthra-
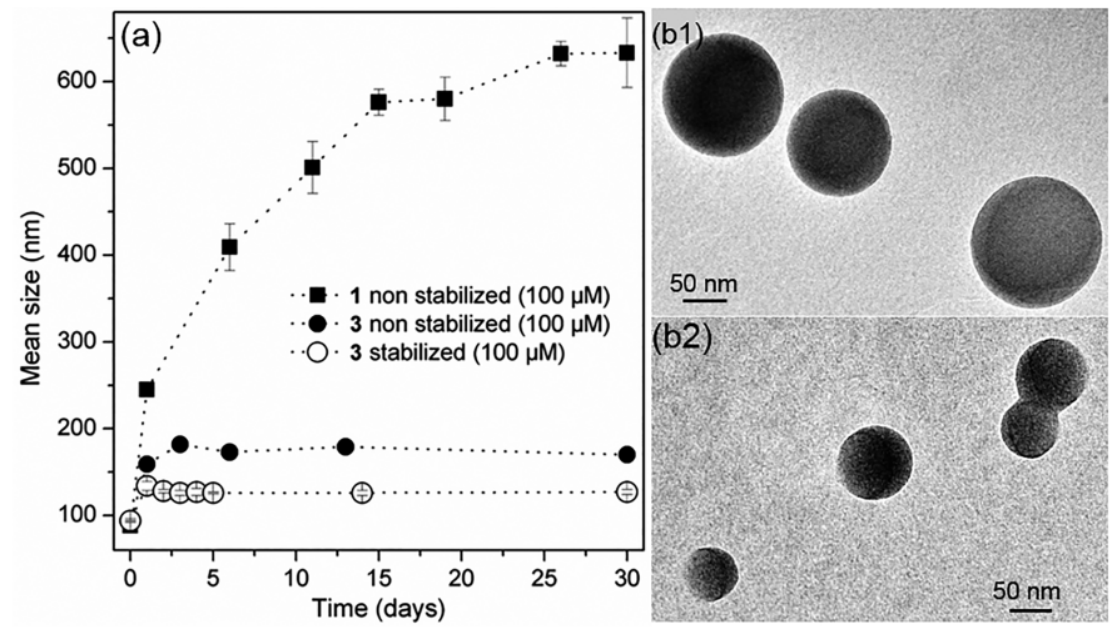

Figure 3. (a) DLS of the supramolecular structures obtained from $\mathbf{1}$ and $\mathbf{3}$ with (stabilized) or without (nonstabilized) ammonia vapors and (b) TEM images of the stabilized spheres obtained from 2 (b1) and 3 (b2) (100 $\mu \mathrm{M}, 90 \%$ water). 

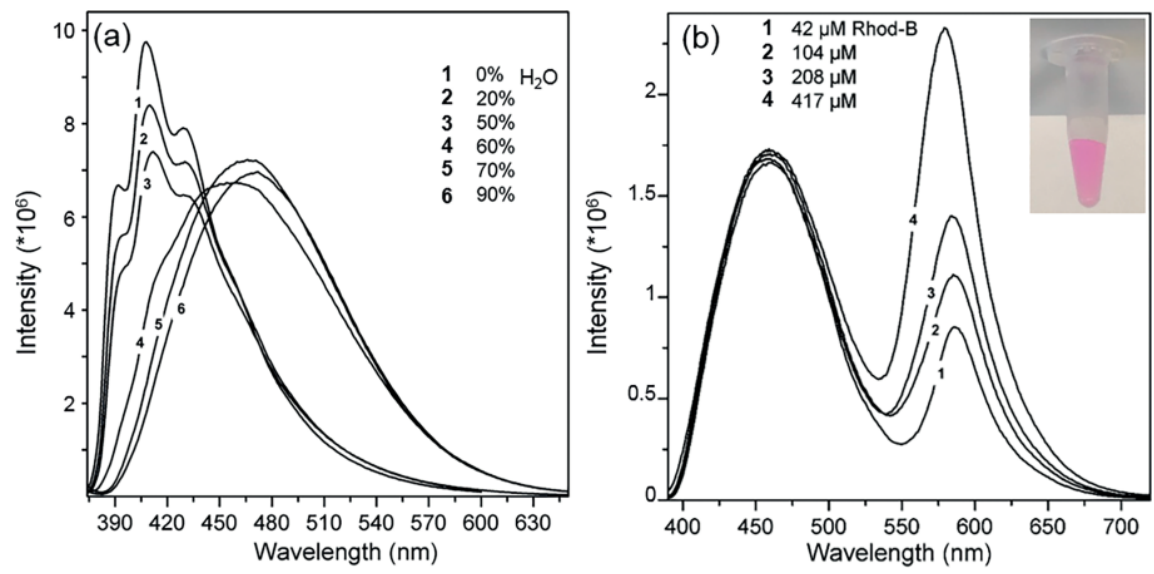

Figure 4. Emission spectra of $\mathbf{3}$ (a) as a function of the water content ( $100 \mu \mathrm{m}, \lambda_{\mathrm{ex}}=375 \mathrm{~nm}$ ) and (b) after the encapsulation of rhod-B at different concentrations ([3] $=200 \mu \mathrm{m}, 90 \%$ water, $\left.\lambda_{\text {ex }}=378 \mathrm{~nm}\right)$.

cene moiety. Different concentrations of rhod-B were added during precipitation of 3 (200 $\mu \mathrm{m}, 90 \%$ water). After stabilization and removal of nonencapsulated dye upon centrifugation, a pink precipitate consisting of hybrid particles incorporating rhod-B was recovered and further redispersed in water (Figure $4 \mathrm{~b}$, inset). DLS measurements showed an increase in size from $90 \mathrm{~nm}$ to ca. $160 \mathrm{~nm}$ upon rhod-B encapsulation, whereas no variation in the $\zeta$ potential could be detected; this was indicative of dye encapsulation rather than surface adsorption (Tables S3 and S4). A photoluminescence study was performed to provide additional evidence for the encapsulation of rhod-B in stabilized hybrid particles of $\mathbf{3}$. The emission spectra recorded at an excitation wavelength in the anthracene region $(378 \mathrm{~nm})$ allowed detection of the emission of both the anthracene (centered at $460 \mathrm{~nm}$ ) and rhod-B (at $580 \mathrm{~nm}$ ) moieties through FRET (Figure $4 \mathrm{~b}$ ). The excitation spectra recorded at the maximum intensity of rhod-B emission $\left(\lambda_{\mathrm{em}}=587 \mathrm{~nm}\right)$ also showed the contribution of the anthracene moiety in $\mathbf{3}$; this confirmed the FRET phenomenon between rhod-B and $\mathbf{3}$ and further evidenced the encapsulation of rhod-B (Figure S5b). Noteworthy, the emission intensity of rhod-B increased with its initial concentration, whereas the emission intensity of $\mathbf{3}$ remained constant (Figure 4b). The ratio of the areas of the emission bands of rhod-B and 3 rapidly increased with the rhod- $B$ concentration before it started to level off around $2 \mathrm{~mm}$, which may have indicated that saturation was approaching (Figure S6). The saturation regime could not be investigated further owing to destruction of the hybrid particles at higher rhod- $B$ concentrations. Importantly, up to $2 \mathrm{~mm}$ rhod-B, the integrity of the particles could be preserved (Figure S7). These results show the robustness of the molecular design, for which stable spherical nanoparticles could still be obtained even after the addition of rhod-B.

\section{Conclusion}

Spherical nanoparticles with well-defined tunable diameters were obtained in a single step by antisolvent precipitation. Our "all-in-one molecule" was based on an organic moiety, a trityl group, which alone was responsible for nanoparticle formation.
The addition of an alkoxysilane group allowed stabilization of the hybrid particles by hydrolysis and condensation so that the final system could be frozen, and further shape and size evolution could be prevented. We showed that the propensity of the trityl group to generate supramolecular spheres provided this molecular design with a unique robustness that allowed the covalent introduction of large organic groups without changing the structural properties of the particles. This opens the door towards a broad range of functional implementations, as illustrated by the covalent conjugation of fluorophores. In addition, such nanoparticles allowed the encapsulation of large molecules without modifying their structure, which indeed opens up additional opportunities. Finally, we have to keep in mind that the functionality of those nanoparticles could be even broader given the versatility of silane chemistry for further functionalization of the nanoparticles' surface, in particular upon envisioning applications in biotechnologies and nanomedicine.

\section{Experimental Section}

Preparation of Hybrid Nanoparticles 1, 2, and 3: A solution of 1, $\mathbf{2}$, or $\mathbf{3}$ in ethanol was quickly added to freshly deionized water under vigorous stirring (always the same speed) at room temperature for $30 \mathrm{~s}$. For each precursor, the water/ethanol ratio (from 0 to $95 \%$ water) and concentration $(100,200$, and $400 \mu \mathrm{M})$ were varied, and the final volume was always kept at $1.5 \mathrm{~mL}$ for reproducibility reasons. For nonstabilized systems, the suspension was left without further stirring. For stabilized systems, the suspension was placed in a sealed environment for $24 \mathrm{~h}$ in the presence of ammonia vapors, which was followed by aging for $48 \mathrm{~h}$ in the newly basified suspension through ammonia transfer. To recover the stabilized hybrid nanoparticles, the suspension was centrifuged and washed with a mixture of ethanol/deionized water (1:1) and then deionized water $(4 \mathrm{X})$

Preparation of Hybrid Nanoparticles 3 Containing Rhodamine B: A solution of $\mathbf{3}$ in ethanol was quickly added to a solution of freshly deionized water ( $90 \%$ water content in all cases) containing $0.2 \mathrm{mg}(42 \mu \mathrm{M}), 0.5 \mathrm{mg}(104 \mu \mathrm{M}), 1 \mathrm{mg}(208 \mu \mathrm{M}), 2 \mathrm{mg}(417 \mu \mathrm{M})$, $5 \mathrm{mg}(834 \mu \mathrm{M})$, or $10 \mathrm{mg}(2080 \mu \mathrm{M})$ of rhodamine $\mathrm{B}$. The conditions of stirring, temperature, volume, stabilization, and washing were the same as those for the preparation of hybrid nanoparticles 3 (see 
above). Although in this case, the final concentration of $\mathbf{3}$ was kept at $200 \mu \mathrm{M}$ only.

Dynamic Light Scattering (DLS) and $\zeta$-Potential Measurements: $\zeta$-potential and size-distribution measurements were performed by using a Malvern Zetasizer Nano ZS90 instrument. The autocorrelation functions were recorded at a scattering angle $\theta=90^{\circ}$ and were analyzed by the non-negatively constrained least-squares technique (NNLS-Multiple Pass) for the determination of particle diameter. All the suspensions were sonicated for $5 \mathrm{~s}$ prior to measurement at $20{ }^{\circ} \mathrm{C}$. For DLS measurements, all measurements were run in triplicate to obtain the size standard deviation.

Transmission Electron Microscopy: A drop of sample in aqueous solution $(7 \mu \mathrm{L})$ was deposited on carbon-coated copper grids (400 mesh, Electron Microscopy Sciences). After $3 \mathrm{~min}$, the excess liquid was blotted with filter paper (Whatman \#4). TEM was performed at room temperature by using a FEI Technai Spirit G2 operating at $120 \mathrm{kV}$. Images were recorded with a Gatan Orius CCD camera.

Fluorescence Spectroscopy: Fluorescence measurements were recorded with a Fluoromax-3 (Horiba Jobin Yvon Perkin-Elmer LS55) spectrometer, with a $150 \mathrm{~W}$ ozone-free xenon arc-lamp as excitation source. Emission spectra (350-650 nm for 3, and 390-725 nm for rhodamine B @ 3) were recorded with excitation wavelengths of 375 and $378 \mathrm{~nm}$ in the absence and presence of rhodamine $\mathrm{B}$, respectively. The bandpass of the excitation and emission monochromators was set at $5 \mathrm{~nm}$, and the scan speed was set at $10 \mathrm{~nm} \mathrm{~s}^{-1}$. Samples were investigated at $25^{\circ} \mathrm{C}$ in $1 \mathrm{~cm}$ path-length cells.

\section{Acknowledgments}

The authors thank Clément Sanchez, François Ribot, and Cédric Boissière for fruitful discussions, Gervaise Mosser for cryoTEM observations and careful reading of the manuscript, Patrick le Griel for his help in electron microscopy, Cristina CoelhoDiogo for solid-state NMR spectroscopy measurements, Mohamed Selmane for PXRD experiments, and the Centre National de la Recherche Scientifique (CNRS) and the Idex PSL (ANR-10IDEX-0001-02 PSL) for funding.

Keywords: Nanoparticles · Self-assembly · Supramolecular chemistry · Alkoxysilanes · Hybrid materials

[1] W. Cai, C.-C. Chu, G. Liu, Y.-X. J. Wáng, Small 2015, 11, 4806-4822.

[2] J. Della Rocca, D. Liu, W. Lin, Acc. Chem. Res. 2011, 44, 957-968.

[3] A. Carné, C. Carbonell, I. Imaz, D. Maspoch, Chem. Soc. Rev. 2011, 40, 291-305.

[4] R.-R. Gao, S. Shi, Y.-J. Li, M. Wumaier, X.-C. Hu, T.-M. Yao, Nanoscale 2017, 9, 9589-9597.

[5] H.-H. Zeng, W.-B. Qiu, L. Zhang, R.-P. Liang, J.-D. Qiu, Anal. Chem. 2016, $88,6342-6348$.

[6] C. Aimé, R. Nishiyabu, R. Gondo, N. Kimizuka, Chem. Eur. J. 2010, 16, 3604-3607.

[7] Y. Liu, K. Kathan, W. Saad, R. K. Prud'homme, Phys. Rev. Lett. 2007, 98, 036102.
[8] W. S. Saad, R. K. Prud'homme, Nano Today 2016, 11, 212-227.

[9] Z. Zhu, Biomaterials 2013, 34, 10238-10248.

[10] Z. Zhu, K. Margulis-Goshen, S. Magdassi, Y. Talmon, C. W. Macosko, J. Pharm. Sci. 2010, 99, 4295-4306.

[11] A. P. Wight, M. E. Davis, Chem. Rev. 2002, 102, 3589-3614.

[12] C. Sanchez, B. Lebeau, F. Chaput, J.-P. Boilot, Adv. Mater. 2003, 15, 19691994.

[13] J. G. Croissant, X. Cattoën, J.-O. Durand, M. Wong Chi Man, N. M. Khashab, Nanoscale 2016, 8, 19945-19972.

[14] A. Arkhireeva, J. N. Hay, J. Mater. Chem. 2003, 13, 3122-3127.

[15] M. Nakamura, K. Ishimura, J. Phys. Chem. C 2007, 111, 18892-18898.

[16] J. Macan, K. Tadanaga, M. Tatsumisago, J. Sol-Gel Sci. Technol. 2010, 53, 31-37.

[17] M. Nakamura, K. Ishimura, Langmuir 2008, 24, 5099-5108.

[18] A. Arkhireeva, J. N. Hay, Chem. Mater. 2005, 17, 875-880.

[19] A. Arkhireeva, J. N. Hay, W. Oware, J. Non-Cryst. Solids 2005, 351, 16881695.

[20] I. Noda, T. Kamoto, M. Yamada, Chem. Mater. 2000, 12, 1708-1714.

[21] F. Baumann, M. Schmidt, B. Deubzer, M. Geck, J. Dauth, Macromolecules 1994, 27, 6102-6105.

[22] I. Noda, M. Isikawa, M. Yamawaki, Y. Sasaki, Inorg. Chim. Acta 1997, 263, 149-152.

[23] A. Arkhireeva, J. N. Hay, J. M. Lane, M. Manzano, H. Masters, W. Oware, S. J. Shaw, J. Sol-Gel Sci. Technol. 2004, 31, 31-36.

[24] M. Nakamura, K. Hayashi, M. Nakano, T. Kanadani, K. Miyamoto, T. Kori, K. Horikawa, ACS Nano 2015, 9, 1058-1071.

[25] S. Dirè, V. Tagliazucca, E. Callone, A. Quaranta, Mater. Chem. Phys. 2011, $126,909-917$.

[26] X. Li, Y. Yang, Q. Yang, J. Mater. Chem. A 2013, 1, 1525-1535.

[27] Q. Wang, Y. Liu, H. Yan, Chem. Commun. 2007, 2339-2341.

[28] F. Dong, W. Guo, S.-W. Chu, C.-S. Ha, Chem. Commun. 2010, 46, 74987500.

[29] F. Dong, W. Guo, S.-S. Park, C.-S. Ha, J. Mater. Chem. 2011, 21, 1074410749.

[30] Y. Xing, J. Peng, K. Xu, W. Lin, S. Gao, Y. Ren, X. Gui, S. Liang, M. Chen, Chem. Eur. J. 2016, 22, 2114-2126.

[31] H. Ujiie, A. Shimojima, K. Kuroda, Chem. Commun. 2015, 51, 3211-3214.

[32] P. Horcajada, A. Rámila, G. Férey, M. Vallet-Regí, Solid State Sci. 2006, 8, 1243-1249.

[33] A. Liberman, N. Mendez, W. C. Trogler, A. C. Kummel, Surf. Sci. Rep. 2014, 69, 132-158.

[34] P. Yang, S. Gaib, J. Lin, Chem. Soc. Rev. 2012, 41, 3679-3698.

[35] D. Kumar, I. Mutreja, P. C. Keshvan, M. Bhat, A. K. Dinda, S. Mitra, J. Pharm. Sci. 2015, 104, 3943-3951.

[36] N. Ornelas-Soto, R. Rubio-Govea, C. E. Guerrero-Beltrán, E. Vázquez-Garza, J. Bernal-Ramírez, A. García-García, Y. Oropeza-Almazán, G. García-Rivas, F. F. Contreras-Torres, Mater. Sci. Eng. C 2017, 79, 831-840.

[37] H. J. Hah, J. S. Kim, B. J. Jeon, S. M. Koo, Y. E. Lee, Chem. Commun. 2003, $1712-1713$.

[38] J. Qian, X. Li, M. Wei, X. Gao, Z. Xu, S. He, Opt. Express 2008, 16, 1956819578.

[39] R. Kumar, I. Roy, T. Y. Hulchanskyy, L. N. Goswami, A. C. Bonoiu, E. J. Bergey, K. M. Tramposch, A. Maitra, P. N. Prasad, ACS Nano 2008, 2, 449456.

[40] J. J. E. Moreau, L. Vellutini, M. Wong Chi Man, C. Bied, J. Am. Chem. Soc. 2001, 123, 1509-1510.

[41] J. J. E. Moreau, L. Vellutini, M. Wong Chi Man, C. Bied, P. Dieudonné, J.-L. Bantignies, J.-L. Sauvajol, Chem. Eur. J. 2005, 11, 1527-1537.

[42] A. Shimojima, Z. Liu, T. Ohsuna, O. Terasaki, K. Kuroda, J. Am. Chem. Soc. 2005, 127, 14108-14116. 